\title{
LIE ALGEBRA MULTIPLICITIES ${ }^{1}$
}

\author{
S. BERMAN AND R. V. MOODY
}

\begin{abstract}
Exact formulas for root space multiplicities in Cartan matrix Lie algebras and their universal enveloping algebras are computed. We go on to determine the number of free generators of each degree of the radicals defining these algebras.
\end{abstract}

1. Introduction. The remarkable product formula

$$
\sum_{w \in W}(-1)^{l(w)} e(s(w))=\prod_{\alpha \in \Delta^{+}}(1-e(\alpha))^{m_{\alpha}}
$$

relating the Weyl group and the roots of an arbitrary Euclidean Lie algebra was discovered and proved by I. G. Macdonald [7] (for notation see below). Subsequently V. G. Kac [4] gave a new proof of this which was shorter and explicitly involved the Lie algebra, and at the same time established that the formula was valid for the entire class of Lie algebras deriving from symmetrizable Cartan matrices. This has been written up in a very lucid way by H. Garland and J. Lepowsky [3], where the formula is obtained by the Euler-Poincaré principle applied to Lie algebra homology.

In the Euclidean case, where the multiplicities $m_{\alpha}$ were independently known, Macdonald used the formula as a powerful tool in producing identities for certain number theoretical functions, notably Dedekind's $\eta$-function. This work has been greatly extended by J. Lepowsky where (1) is used to prove combinatorial expansions for every positive power of $\eta$ [6]. In other cases it serves as a method, indeed the only one known, for computing the multiplicities. Up to now one has used the formula as it stands, computing the multiplicities inductively. Here we show how to invert the formula. We obtain an explicit formula (Theorem 2) for the multiplicities which can be seen as an analogue of Witt's dimension formula for free Lie algebras.

The Lie algebras $\mathcal{E}$ under consideration (see [5] or [9]) are factors of a well-understood graded Lie algebra $\tilde{\mathcal{L}}$ (see [1]) by a homogeneous ideal $R, \tilde{\mathfrak{L}}$ is the direct sum $\tilde{\mathfrak{L}}^{-} \oplus \tilde{\mathcal{H}} \oplus \tilde{\mathfrak{L}}^{+}$where $\tilde{\mathfrak{L}}^{-}$and $\tilde{\mathfrak{L}}^{+}$are isomorphic free Lie algebras and $\mathcal{H}$ is abelian and acts diagonally. $R$ splits up accordingly: $R=R^{+} \oplus R^{0} \oplus R^{-}$, and $R^{+}$and $R^{-}$are themselves ideals of $\mathcal{L}^{+}$and $\mathcal{L}^{-}$. These are free Lie algebras and possess a free generating system consisting of homogeneous elements [2]. In Theorem 3 we obtain a formula for the number

Received by the editors September 28, 1978.

AMS (MOS) subject classifications (1970). Primary 17B65, 17B35.

${ }^{1}$ Both authors were assisted in this work by National Research Council of Canada operating grants. 
of free generators of each degree in such a system.

2. Notation. The notation here is consistent with that of [8], in which one can find all the relevant definitions. $\Delta$ is the root system of a symmetrizable Cartan matrix $\left(A_{i j}\right)$ and $\Delta^{+}$is the set of positive roots of $\Delta$ relative to some base $\left\{\alpha_{1}, \ldots, \alpha_{l}\right\}$ of $\Delta .(Z \Delta)^{+}$is the set of nonnegative integral linear combinations of $\alpha_{1}, \ldots, \alpha_{l}$ and for $\beta=\Sigma z_{i} \alpha_{i} \in(Z \Delta)^{+}$, the height ht $\beta$ of $\beta$ is $\Sigma z_{i} . W$ is the Weyl group and for each $w \in W, l(w)$ is the length of $w$ as an expression of minimum length in terms of the fundamental reflections $r_{1}, \ldots, r_{l}$ in $\alpha_{1}, \ldots, \alpha_{l}$. For each $w \in W, s(w)$ is the sum of the positive roots which are mapped into $-\Delta^{+}$by $w^{-1}$. For $\alpha \in(\mathbf{Z} \Delta)^{+}$the multiplicity $m_{\alpha}$ of $\alpha$ is the dimension of the corresponding root space. It is taken to be 0 if $\alpha$ is not a root. The $e(\alpha)$ are formal exponentials, which means that we construct the integral semigroup algebra of the semigroup $\left((Z \Delta)^{+},+\right)$, in which we treat $(\mathrm{Z} \Delta)^{+}$multiplicatively letting $\alpha$ be denoted by $e(\alpha)$. Thus the $e(\alpha)\left(\alpha \in(\mathrm{Z} \Delta)^{+}\right)$are all Z-independent and $e(\alpha) e(\beta)=e(\alpha+\beta)$. In formulas such as (1) the sums and products are formal, being taken in order of increasing height.

The universal enveloping algebras $U\left(\mathfrak{L}^{+}\right)$and $U\left(\tilde{\mathfrak{E}}^{+}\right)$inherit the grading of $\mathfrak{L}^{+}$and $\tilde{\mathfrak{L}}^{+}$by $(\mathrm{Z} \Delta)^{+}$. For $\alpha \in(\mathbf{Z} \Delta)^{+}, n_{\alpha}$ and $\tilde{n}_{\alpha}$ denote the dimensions of the spaces $U\left(\mathfrak{L}^{+}\right)_{\alpha}$ and $U\left(\tilde{\mathfrak{L}}^{+}\right)_{\alpha}$. Let $\tilde{m}_{\alpha}=\operatorname{dim} \tilde{\mathfrak{L}}_{\alpha}$.

Let $s_{0}, s_{1}, s_{2}, \ldots$ be the set of elements $s(w), w \in W$, written in an order of increasing height. Thus $s_{0}=s(1)=0$ (empty sum) and $s_{1}, s_{2}, \ldots, s_{l}$ are $\alpha_{1}, \ldots, \alpha_{l}$ in some order. For each $s(w)$ let $\varepsilon(s(w))=-(-1)^{l(w)}$. In this notation (1) assumes the form

$$
1-\sum_{i=1}^{\infty} \varepsilon\left(s_{i}\right) e\left(s_{i}\right)=\prod_{\alpha \in \Delta^{+}}(1-e(\alpha))^{m_{\alpha}} .
$$

Now introduce partitions of elements of $(\mathrm{Z} \Delta)^{+}$into sums of the $s_{i}$. For each sequence $(n)=\left(n_{1}, n_{2}, n_{3}, \ldots\right)$ of nonnegative integers $n_{i}$, all but a finite number being zero, consider $\Sigma n_{i} s_{i} \in(\mathrm{Z} \Delta)^{+}$(note that $s_{0}$ is not included in this). For $\lambda \in(\mathrm{Z} \Delta)^{+}, S(\lambda)$ is defined to be $\left\{(n) \mid \sum n_{i} s_{i}=\lambda\right\}$. We write $\Sigma(n)$ for $\sum_{i=1}^{\infty} n_{i}, B(n)$ for $B((n)):=\left(\sum n_{i}\right) ! / \Pi\left(n_{i} !\right)$, and $\operatorname{sgn}(n)$ for $\operatorname{sgn}((n)):=\Pi \varepsilon\left(s_{i}\right)^{n_{1}}$. For $\alpha, \lambda \in(Z \Delta)^{+}$we write $\lambda \mid \alpha$ if $\alpha=r \lambda$ for some positive integer $r$ and denote $1 / r$ by $\lambda / \alpha$. Finally, $\mu$ denotes the Möbius function.

\section{The formulas.}

THEOREM 1. For all $\alpha \in(\mathbf{Z} \Delta)^{+}$,

$$
n_{\alpha}=\sum_{(n) \in S(\alpha)} \operatorname{sgn}(n) B(n) .
$$

Proof. Using the Poincaré-Birkhoff-Witt theorem followed by the Macdonald-Kac identity $\left(1^{\prime}\right)$ we have 


$$
\begin{aligned}
\sum_{\alpha \in(\mathrm{Z} \Delta)^{+}} n_{\alpha} e^{\alpha} & =\frac{1}{\prod_{\alpha \in \Delta}(1-e(\alpha))^{m_{\alpha}}} \\
& =\frac{1}{1-\sum_{i=1}^{\infty} \varepsilon\left(s_{i}\right) e\left(s_{i}\right)}=\sum_{k=0}^{\infty}\left(\sum_{i=1}^{\infty} \varepsilon\left(s_{i}\right) e\left(s_{i}\right)\right)^{k} \\
& =\sum_{\alpha \in(\mathbf{Z} \Delta)^{+}(n) \in S(\alpha)} \sum \operatorname{sgn}(n) B(n) e(\alpha) .
\end{aligned}
$$

TheOREM 2. For all $\alpha \in(\mathrm{Z} \Delta)^{+}, \alpha \neq 0$,

$$
m_{\alpha}=\sum_{\lambda \mid \alpha} \mu\left(\frac{\alpha}{\lambda}\right) \frac{\lambda}{\alpha} \sum_{(n) \in S(\lambda)}\left(\Pi \varepsilon\left(s_{i}\right)^{n_{i}}\right) \frac{\left(\left(\sum n_{i}\right)-1\right) !}{\Pi\left(n_{i} !\right)} .
$$

Proof. Let the left-hand side of (1) be denoted by $\Sigma$. Applying $-\log$ to (1')

$$
\begin{aligned}
-\log \Sigma & =\sum_{\alpha \in \Delta^{+}} m_{\alpha}(-\log (1-e(\alpha))) \\
& =\sum_{\alpha \in(\mathbf{Z} \Delta)^{+}} m_{\alpha} \sum_{k=1}^{\infty} \frac{e(\alpha)^{k}}{k}=\sum_{\alpha, k} m_{\alpha} \frac{e(k \alpha)}{k} .
\end{aligned}
$$

Apply the operator

$$
E=\sum_{i=1}^{l} e\left(\alpha_{i}\right) \frac{\partial}{\partial e\left(\alpha_{i}\right)}
$$

to get

$$
-\frac{E(\Sigma)}{\Sigma}=\sum_{\alpha, k} m_{\alpha} \frac{h t(k \alpha)}{k} e(k \alpha)
$$

Set

$$
C(\lambda)=\sum_{\alpha \mid \lambda} m_{\alpha} h t \alpha .
$$

so the right-hand side of (2) becomes $\Sigma_{\lambda \in(\mathrm{Z} \Delta)^{+}} C(\lambda) e(\lambda)$. Now

$$
-E(\Sigma)=\sum_{i=0}^{\infty} \varepsilon\left(s_{i}\right) \mathrm{ht}\left(s_{i}\right) e\left(s_{i}\right)
$$

and

$$
\Sigma^{-1}=\sum_{\alpha \in(\mathbb{Z} \Delta)^{+}} \sum_{(n) \in S(\alpha)} \operatorname{sgn}(n) B(n) e(\alpha)
$$

(see Theorem 1). Thus

$$
\frac{-E(\Sigma)}{\Sigma}=\sum_{i=0}^{\infty} \varepsilon\left(s_{i}\right) \mathrm{ht}\left(s_{i}\right) e\left(s_{i}\right) \cdot \sum_{\alpha \in(\mathbf{Z} \Delta)^{+}} \sum_{(n) \in S(\alpha)} \operatorname{sgn}(n) B(n) e(\alpha) .
$$

The coefficient of $e(\lambda)$ in (4) is

$$
\sum_{i=1}^{\infty} \varepsilon\left(s_{i}\right) \operatorname{ht}\left(s_{i}\right) \sum_{(n) \in S\left(\lambda-s_{i}\right)} \operatorname{sgn}(n) B(n)
$$


[note. that ht $\left(s_{0}\right)=0$ ]. Evidently if $(n)=\left(n_{1}, n_{2}, \ldots\right) \in S\left(\lambda-s_{i}\right)$ then

$$
\left(n_{1}, n_{2}, \ldots, n_{i}+1, \ldots\right) \in S(\lambda)
$$

conversely if $\left(n_{1}, n_{2}, \ldots\right) \in S(\lambda)$ then for each $i$ for which $n_{i}>0$,

$$
\left(n_{1}, n_{2}, \ldots, n_{i}-1, \ldots\right) \in S\left(\lambda-s_{i}\right) \text {. }
$$

Thus each partition $(n) \in S(\lambda)$ makes a contribution of size

$$
\varepsilon\left(s_{i}\right) \operatorname{ht}\left(s_{i}\right) \operatorname{sgn}\left((n)^{(i)}\right) B\left((n)^{(i)}\right)
$$

in (5) once for each $i$ for which $n_{i}>0$, where

$$
(n)^{(i)}:=\left(n_{1}, n_{2}, \ldots, n_{i}-1, \ldots\right)
$$

Since

and

$$
\begin{gathered}
B\left((n)^{(i)}\right)=\left(\left(\sum n_{j}\right)-1\right) ! n_{i} / \Pi\left(n_{j} !\right), \\
\varepsilon\left(s_{i}\right) \operatorname{sgn}\left((n)^{(i)}\right)=\operatorname{sgn}(n),
\end{gathered}
$$

$$
\sum n_{i} \mathrm{ht}\left(s_{i}\right)=\mathrm{ht}(\lambda),
$$

there is a total contribution of $\operatorname{ht}(\lambda) \operatorname{sgn}(n)\left(\left(\sum n_{j}\right)-1\right) ! / \Pi\left(n_{j} !\right)$. Thus

$$
C(\lambda)=\operatorname{ht}(\lambda) \sum_{(n) \in S(\lambda)} \operatorname{sgn}(n)\left(\left(\Sigma n_{i}\right)-1\right) ! / \Pi\left(n_{i} !\right) .
$$

The theorem follows by Möbius inversion of (3).

In spite of its apparent complexity the formula is quite effective, especially at low heights, since the $s_{i}$ rise rapidly in height and relatively few are involved in any particular case.

In the limiting case when all the off-diagonal entries of the Cartan matrix are $\infty$, the part of the Lie algebra spanned by the positive root spaces is the free Lie algebra on $l$ generators. Then there are no $s_{i}$ 's past $i=l$ and for each $\lambda=\sum n_{i} \alpha_{i} \in(\mathbf{Z} \Delta)^{+}, S(\lambda)$ reduces to the single partition $(n)=\left(n_{1}, n_{2}, \ldots, n_{l}\right)$. The multiplicity formula then collapses to the well-known Witt formula.

Recall that $\mathfrak{L}^{+}=\tilde{\mathfrak{L}}^{+} / R^{+}$and $R^{+}$is a homogeneous ideal of $\tilde{\mathfrak{L}}^{+}$. Let $r_{\alpha}=\operatorname{dim} R_{\alpha}^{+}, \alpha \in(\mathbf{Z} \Delta)^{+}$. Since $\tilde{\mathfrak{L}}^{+}$is free, it is well known that $R^{+}$is a free Lie algebra. One also knows [2, Chapter II, \$2, Problem 13, p. 184] that $R^{+}$ has a set of homogeneous free generators. Let $g_{\alpha}$ denote the number of these generators of degree $\alpha$. Finally let $u_{\alpha}$ be the dimension of the space of elements of degree $\alpha$ in the universal enveloping algebra $U\left(R^{+}\right)$of $R^{+}$. We view $U\left(R^{+}\right)$as a subalgebra of $U\left(\tilde{\mathfrak{E}}^{+}\right)$.

THEOREM 3. For all $\alpha \in(\mathrm{Z} \Delta)^{+}, \alpha \neq 0$,

$$
g_{\alpha}=-\sum_{(n) \in S(\alpha)} \operatorname{sgn}(n) B(n)\left(1-\frac{n_{1}+\cdots+n_{l}}{\sum(n)}\right) .
$$


Proof. $U\left(R^{+}\right)$is a free associative algebra freely generated by the generators of the free Lie algebra $R^{+}$. It follows that

$$
\frac{1}{1-\sum g_{\alpha} e(\alpha)}=\sum u_{\alpha} e(\alpha)
$$

On the other hand

$$
\sum u_{\alpha} e(\alpha)=\frac{1}{\Pi(1-e(\alpha))^{r_{\alpha}}}
$$

by the Poincaré-Birkhoff-Witt theorem.

Combining the two equalities we have

$$
\begin{aligned}
1-\sum g_{\alpha} e(\alpha)= & \Pi(1-e(\alpha))^{r_{\alpha}}=\frac{\Pi(1-e(\alpha))^{\tilde{m}_{\alpha}}}{\Pi(1-e(\alpha))^{m_{\alpha}}} \\
= & \left(1-\left[e\left(\alpha_{1}\right)+\cdots+e\left(\alpha_{l}\right)\right]\right) \\
& \cdot \sum_{\alpha \in(\mathbf{Z} \Delta)^{+}} \sum_{(n) \in S(\alpha)} \operatorname{sgn}(n) B(n) e(\alpha),
\end{aligned}
$$

the last equality following from the formula

$$
\frac{1}{1-\left[e\left(\alpha_{1}\right)+\cdots+e\left(\alpha_{l}\right)\right]}=\sum \tilde{n}_{\alpha} e(\alpha)=\frac{1}{\Pi(1-e(\alpha))^{\tilde{m}_{\alpha}}}
$$

and Theorem 1. We conclude that for $\alpha \in(\mathbf{Z} \Delta)^{+}, \alpha \neq 0$

$$
-g_{\alpha}=\sum_{(n) \in S(\alpha)} \operatorname{sgn}(n) B(n)-\sum_{i=1}^{l} \sum_{(n) \in S\left(\alpha-\alpha_{i}\right)} \operatorname{sgn}(n) B(n) .
$$

Every $(n) \in S(\alpha)$ with $n_{i}>0$ determines

$$
\left(n^{\prime}\right)=\left(n_{1}, \ldots, n_{i}-1, \ldots\right) \in S\left(\alpha-\alpha_{i}\right)
$$

whose contribution to the sum over $S\left(\alpha-\alpha_{i}\right)$ is $\operatorname{sgn}(n) n_{i} B(n) / \Sigma(n)$ (note $\varepsilon\left(s_{i}\right)=1$ for $\left.i=1, \ldots, l\right)$. This is evidently valid even if $n_{i}=0$, and every $(m) \in S\left(\alpha-\alpha_{i}\right), i=1, \ldots, l$, is accounted for in this way. Thus

$$
-g_{\alpha}=\sum_{(n) \in S(\alpha)} \operatorname{sgn}(n) B(n)\left[1-\sum_{i=1}^{l} \frac{n_{i}}{\sum(n)}\right] .
$$

\section{REFERENCES}

1. S. Berman, Isomorphisms and automorphisms of universal Heffalump Lie algebras, Proc. Amer. Math. Soc. 65 (1977), 29-34.

2. N. Bourbaki, Lie groups and Lie algebras, Part 1, Chapters 1-3, Addison-Wesley, Reading, Mass., 1975.

3. H. Garland and J. Lepowsky, Lie algebra homology and the Macdonald-Kac formula, Invent. Math. 34 (1976), 37-76.

4. V. G. Kac, Infinite-dimensional Lie algebras and Dedekind's $\eta$-function, Funkcional. Anal. i Priložen. 8 (1974), 77-78; English transl., Functional Anal. Appl. 8 (1974), 68-70. 
5. 1271-1311.

6. J. Lepowsky, Macdonald type identities, Advances in Math. 27 (1978), 230-234.

7. I. G. Macdonald, Affine root systems and Dedekind's $\eta$-function, Invent. Math. 15 (1972), 91-143.

8. R. V. Moody, Root systems of hyperbolic type, Advances in Math. (to appear).

9. ___ A new class of Lie algebras, J. Algebra 10 (1968), 211-230.

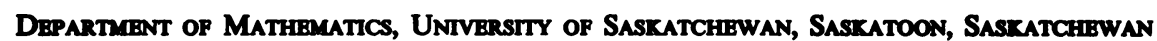
STN OWO, CANADA 